$$
F_{r}^{\mathrm{MO}}=N_{\max }-N_{r}, \text { wobei } N_{r}=\sum_{s}^{\prime} p_{r s} .
$$

$N_{r}$ mißt die Bindungsbeanspruchung des Atoms $r$ im Molekül, der maximal mögliche Wert $N_{\max }$ von $N_{r}$ ist nach Moffitt ${ }^{13} \sqrt{3}, \sqrt{2}$ oder $\sqrt{1}$, je nachdem ob das betrachtete C-Atom mit 3, 2 oder 1 trigonalen C-Atomen verknüpft ist. Der Anteil 3, 2 oder 1 der $\sigma$-Bindungen ist darin - wie in allen Formeln dieser Arbeit - nicht enthalten, er würde sich bei der Differenzbildung in (8) auch herausheben.

(8) gilt für gerade und ungerade Elektronenzahl und stimmt mit (7) bis auf eine additive Konstante überein. Es ist jedoch zu beachten, daß (8) nicht aus der ursprünglichen Definition (4) abgeleitet werden kann, da in der MO-Methode keine (1) oder (2) entsprechende Beziehung zu bestehen scheint. Da ferner - wie schon erwähnt - die nach beiden Methoden berechneten $p_{r s}$ nicht unmittelbar vergleichbar sind, gilt dies auch für die $F_{r}$, insbesondere für deren Absolutwerte. Der relative Verlauf von $F_{r}$ für die verschiedenen Positionen eines Moleküls ist jedoch in beiden Methoden im allgemeinen ähnlich.

Zur Erläuterung der obigen Überlegungen und Begriffe seien zum Schluß freie Valenzen für einige Moleküle angegeben.

${ }^{13}$ W. Moffitt, Fußnote zu C. A. Coulson, J. Chim. Phys. 45, 243 [1948].
1. Äthylen: Die $\pi$-Bindungsordnung der $\mathrm{C}=\mathrm{C}$ Bindung ist gleich 1 und daher nach (7) $F_{1}=F_{2}=0$.

2. Benzol: $p=0,623$ für alle Bindungen ${ }^{3}$, also ist $F=1-2 \cdot 0,623=-0,246$.

\section{Allylradikal:}

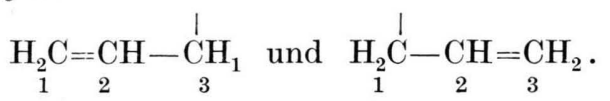

(I, 12) oder (I, 15) gibt die Bindungsordnung $p_{12}=p_{23}=2 / 3$ und nach (7) wird $F_{1}=F_{3}=1 / 3$, $F_{2}=-1 / 3$.

Die beiden Endatome ergeben sich also als reaktionsfähiger als das Zentralatom in Übereinstimmung mit der Erfahrung. Moffitt ${ }^{5}$ erhält für seine „residual affinity" für die Endatome den Wert $+2 / 3$, für das Zentralatom den gleichen Wert - $1 / 3$ wie oben.

4. Naphthalin: Hier muß der relative Gang der freien Valenzen das höhere Reaktionsvermögen der $\alpha$-Position gegenüber der $\beta$-Position wiedergeben. Mit den Penneyschen Werten für die Bindungsordnungen ${ }^{3}$ erhält man auch $F_{\alpha}=-0,206, F_{\beta}=$ $-0,220$.

Allgemein erhält man für olefinische C-Atome Werte um Null, für aromatische C-Atome Werte in der Gegend um -0,25, während positive Werte von $F_{r}$ für freie Radikale charakteristisch sind.

\title{
Zur Rolle der pythagoreischen Metrik in der Physik
}

\author{
Von Detle f Laugwitz* \\ Aus dem Mathematischen Institut der Universität Göttingen \\ (Z. Naturforschg. 9a, 827-832 [1954]; eingegangen am 31. Juli 1954)
}

\begin{abstract}
Es wird eine Kennzeichnung der Räume mit pythagoreischer Metrik angegeben, welche über die Ergebnisse von Helm holtz und W eyl insofern hinausgeht, als außer dem euklidischen und Riemannschen Raum der klassischen und relativistischen Physik auch die quadratische Metrik im quantenmechanischen Zustandsraum erfaßt wird. Die Kennzeichnung beruht auf einem Isotropiepostulat, das im Zustandsraum in die Forderung der Gleichberechtigung aller Zustände vor der Beobachtung übergeht.
\end{abstract}

$\mathrm{D}^{\mathrm{s}}$ e bisherigen Versuche (v. Helmholtz, Weyl), die pythagoreische Struktur des Raumes der Physik auf eine grundlegende Eigenschaft zurückzuführen, sind, wie v. We i z ä c k e ${ }^{1}$ gezeigt hat, vom heutigen Standpunkt nicht voll befriedigend. Insbesondere sollte mit berücksichtigt werden, daß

\footnotetext{
* Göttingen, Nußanger 70.
}

auch in der Gesamtheit der Zustände eines quantenmechanischen Systems eine quadratische Metrik eine wesentliche Rolle spielt, und es wird vorgeschlagen, die pythagoreische Metrik mittels einer Eigenschaft zu begründen, welche diesen Gegebenheiten gemeinsam ist. In der vorliegenden Note soll

1 C. F. v. Weizsäcker, Z. Naturforschg. 7 a, 141 [1952]. 
der Versuch unternommen werden, diese Begründung mit Hilfe einer geometrischen Grundeigenschaft durchzuführen, welche von dem gemeinsamen Ausgangspunkt Helmholtz' und Weyls, der kontinuierlichen Bewegungsgruppe, wesentlich verschieden ist.

Schon Riemann hat in seiner berühmten Habilitationsvorlesung ${ }^{2}$ das Bedürfnis zum Ausdruck gebracht, die Beschränkung seiner Untersuchung auf metrische Fundamentalformen pythagoreischer Natur zu begründen, nachdem er vorher selbst darauf hingewiesen hat, daß $d s=F(x ; \mathrm{d} x)$ mit positiv-definitem, in den Differentialen von erster Ordnung positiv homogenem $F$ zunächst als natürlicher Ansatz sich darböte. Die Taylorsche Entwicklung von $\mathrm{d} s^{2}$ nach $\mathrm{d} x$ beginnt mit in $\mathrm{d} x$ quadratischen Gliedern, und Riemann betrachtet nur diesen quadratischen Anteil unterVernachlässigung der Glieder höherer Ordnung. Wenn man auf die Riemannsche Begründung auch nicht viel Gewicht legen wird ${ }^{3}$, so ist es doch das Verdienst Riemanns, auf das in Rede stehende Problem erstmals hingewiesen zu haben. Er hat auch bereits angegeben: „,Setzt man . . . wie Euklid . . . eine von der Lage unabhängige Existenz ... der Körper voraus, so folgt, da $\beta$ das Krümmungsma $\beta$ allenthalben konstant ist ..."

v. Helmholtz ${ }^{4}$ macht diese Voraussetzung der freien Beweglichkeit für infinitesimale Körper und beweist dann unter Benützung des Monodromieaxioms, daß der Raum eine Riemannsche Metrik trägt. v. Weizsäcker weist darauf hin, daß die dabei benutzte Existenz starrer und kontinuierlich zusammenhängender Körper vom Standpunkt der heutigen Physik nicht mehr als plausible Grundforderung erscheinen kann und daß sich daher das Raumproblem heute wieder neu stellt.

Weyl $1^{5}$ hat eine Kennzeichnung der Riemannschen Räume angegeben, welche ebenfalls wesentlich auf dem Begriff der Bewegungsgruppe beruht. Er geht von folgendem Prinzip aus: ,,Welche quantitative Ausgestaltung auch immer im Rahmen der Natur der Metrik das metrische Feld gefunden haben mag, stets determiniert das metrische Feld eindeutig den affinen Zusammenhang". Zum Begriff der Metrik wird bemerkt, daß die Kongruenz rein infinitesimal zu fassen sei. Dazu dient einmal die Gruppe der Drehungen um den Punkt $\mathrm{P}_{0}$, zum anderen die Gesamtheit der kongruenten $\mathrm{Ab}$ bildungen des ,Vektorkörpers in $\mathrm{P}_{0}$ “ auf die Vektorkörper in einer infinitesimalen Umgebung von $\mathrm{P}_{0}$. In beiden Fällen wird die Linearität der Abbildungen ohne weitere Begründung verlangt, und dies ist wohl

2 Über die Hypothesen, welche der Geometrie zugrunde liegen, 1854, von H. Weyl kommentierte Neuausgabe, Berlin 1919.

${ }^{3}$ Man vergleiche dazu $H$. Weyl, Mathematische Analyse des Raumproblems, Berlin 1923, S. 29.

${ }^{4}$ H. v. Helmholtz, Über die Tatsachen, die der Geometrie zugrunde liegen, Gött. Nachr. XV [1868]; S. Lie, Theorie der Transformationsgruppen III, Leipzig 1893, S. 394. An neueren Arbeiten seien die jedenfalls für die Drehungsgruppe nicht ohne weiteres durch natürliche Forderungen zu erreichen. Man kann sich dabei nicht darauf berufen, da $\mathrm{B}$ im Infinitesimalen alles linear sein müsse; das wäre ein Argument von der gleichen Art wie das bei der Auslegung von Riemanns Beschränkung auf das quadratische Glied in der Entwicklung von $\mathrm{d} s^{2}$ auftretende. In beiden Fällen werden nämlich im Infinitesimalen Anteile vernachlässigt, die von gleicher Größenordnung wie der Rest sind. Die Drehungen sollen ja nur dazu dienen, die Längen von solchen Vektoren zu vergleichen, die von $P_{0}$ aus aufgetragen sind. Wenn man die Drehungen dann [im ursprünglichen Riemannschen Ansatz, d. h. bei $\mathrm{d} s=F(x ; \mathrm{d} x)]$ für festes $x$ (entsprechend $\mathrm{P}_{0}$ ) unter denjenigen kontinuierlichen Transformationen der $\mathrm{d} x$ sucht, welche den Wert von $F$ nicht verändern und dabei jeden Strahl durch $\mathrm{P}_{0}$, d. h. $\mathrm{d} x=0$, wieder in einen solchen überführen, dann wird man dem von Weyl geforderten infinitesimalen Charakter der Kongruenz völlig gerecht werden. Aber diese Transformationen sind im allgemeinen nicht linear, und die Linearität erscheint von diesem Standpunkt als eine formale Zusatzforderung, auf die man, wenn möglich, gerne verzichten wird. Bei Weyl ist auch nicht zu sehen, unter welchen Räumen die im kleinen euklidischen durch das Postulat ausgezeichnet werden. In dieser Richtung hat Scheibe ${ }^{6}$ die Weylschen Untersuchungen kürzlich ergänzt; es hat sich dabei gezeigt, daß der Ansatz algebraisch sehr ausbaufähig ist, daß andererseits jedoch gerade wegen der wesentlichen $\mathrm{Be}$ schränkung auf lineare Gruppen die für den konkreten Raum in Frage kommenden Maßbestimmungen $\mathrm{d} s=$ $F(x ; \mathrm{d} x)$ nicht zur Konkurrenz zugelassen werden. Das Weylsche Postulat involviert das Verhältnis von $P_{0}$ zu den Punkten einer infinitesimalen Umgebung, und ob die Eindeutigkeitsforderung selbst von so grundlegender Bedeutung ist, scheint nicht evident (vgl. dazu 1. c. ${ }^{1}$ ).

Wir wollen daher hier versuchen, eine Kennzeichnung der Räume mit pythagoreischer Metrik zu geben, welche folgende Forderungen erfüllt:

(A) Die Bedeutung der quadratischen Form in klassisch-relativistischer Geometrie und Quantenmechanik soll aus einem gemeinsamen Prinzip begründet werden.

Und speziell für die klassische Fragestellung von Riemann-Helmholtz-Weyl werden wir verlangen:

(B) Es sollen physikalische Annahmen vermieden werden, welche über die Kontinuität des Rau-

rein geometrische Note von G. Birkhoff, Trans. Amer. Math. Soc. 55, 465 [1944] und G. Pickert, Math. Ann. 120, 492 [1947-49], erwähnt.

${ }^{5}$ H. Weyl, Math. Z. 12, 114 [1921] und 1. c. ${ }^{3}$; eine Behandlung des Weylschen Raumproblems unter sehr allgemeinen Voraussetzungen (Faserräume) führt E. Scheibe in seiner Göttinger Dissertation (1954) durch.

${ }^{6}$ E. Scheibe, l. c. ${ }^{5}$. 
mes und die Existenz einer Metrik im Infinitesimalen hinausgehen.

Die beiden in B positiv gestellten Forderungen an den Raum werden bei Helmholtz und Weyl auch erhoben. Die Einteilung der Vektoren in Klassen ,gleich langer" ist bei Helmholtz im Monodromieaxiom enthalten; dieser Sachverhalt kommt in der Darstellung von Pickert ${ }^{4}$ besonders deutlich zum Ausdruck. Wir verlangen also physikalisch jedenfalls nicht mehr als Helmholtz und Weyl, werden aber darüber hinaus auf gruppentheoretische Forderungen ganz verzichten, da deren physikalische Bedeutung nicht in evidenter Weise fundamental sein mag. Auch ein Bezug auf benachbarte Punkte wird nicht eingehen, wir werden also - wie Weyl es verlangt - rein infinitesimal vorgehen.

Wir kommen jetzt zur genauen Formulierung unserer Voraussetzungen. Die ersten beiden decken sich mit dem ursprünglichen, oben erwähnten Ansatz Riemanns, sollen jedoch im Hinblick auf den für die Quantenmechanik interessanten unendlichdimensionalen Fall ,dimensionsfrei" formuliert werden.

$l^{\prime}$. Der Raum ist eine differenzierbare Mannigfaltigkeit im Sinne der Differentialgeometrie. D. h. genauer: Der Raum ist eine mit einer Hausdorffschen Topologie ausgestattete Menge, und er läßt sich von Untermengen überdecken, die homöomorph zu offenen Teilmengen eines linearen topologischen „Koordinatenraumes" sind, so daß Koordinatentransformationen differenzierbar $\operatorname{sind}^{7}$.

Dies läuft dann bekanntlich darauf hinaus, daß der Raum im kleinen linear ist, und da wir eine rein infinitesimale Kennzeichnung geben wollen, können wir uns auf die Betrachtung des linearen Tangentialraumes (der Menge der kontravarianten Vektoren) in einem beliebigen, aber festgehaltenen Punkte der Mannigfaltigkeit beschränken. Dies hat auch den Vorteil, daß wir den linearen Raum der quantenmechanischen Zustände bequem mit erfassen können; freilich werden wir zunächst nur von reellen Räumen sprechen, doch wird sich das Ergebnis dann leicht auf komplexe Räume erweitern lassen. Wir fordern:

\footnotetext{
7 Eine genauere Erörterung des Begriffs der differenzierbaren Mannigfaltigkeit findet man für die endlichdimensionale Geometrie bei Veblen $\mathrm{u}$. White h ead, Foundations of differential geometry, Cambridge 1932, für den Fall beliebiger Dimension in der Göttinger
}

1. Der Raum ist ein reeller Vektorraum beliebiger, auch unendlicher Dimension (Vektoren $\mathfrak{x}, \mathfrak{y}, \ldots$ ).

2. Im Raum ist eine invariante Funktion $F(\mathfrak{x})$ definiert mit

a) $F(\mathfrak{x})>0$ für $\mathfrak{x} \neq 0$;

b) $F(\lambda \cdot \mathfrak{x})=|\lambda| \cdot F(\mathfrak{x})$ für alle $\mathfrak{x}$ und alle reellen $\lambda$;

c) $F(\mathfrak{x})$ ist stetig in $\mathfrak{x}$ in jedem zweidimensionalen Unterraum.

3. (Isotropiepostulat). Alle Richtungen sind geometrisch gleichberechtigt. (D. h.: Jede geometrische Eigenschaft, die einem vom Nullpunkt ausgehenden Halbstrahl zukommt, trifft auch für jeden anderen zu; geometrisch heißt dabei eine Eigenschaft, die gegenüber Koordinatentransformationen invariant ist, und da im Tangentialraum eine beliebige Koordinatentransformation der Mannigfaltigkeit sich als eine den Nullpunkt festlassende lineare Koordinatentransformation äußert, bedeutet das: Invarianz gegenüber dieser zentrisch-affinen Gruppe. Wir werden das Isotropiepostulat nur in einer speziellen Form benötigen: In der von zwei Richtungen aufgespannten Ebene soll jede geometrische Eigenschaft der einen Richtung auch der anderen Richtung zukommen.)

Für den Fall des ,,physischen Raumes, des Ordnungsschemas der wirklichen Dinge" (Weyl) ist zur Begründung der Postulate zunächst folgendes zu bemerken. Postulat $\mathbf{l}^{\prime}$ und damit dessen Folge 1 besagen nicht mehr, als daß der Raum ein Kontinuum ist, das man im kleinen auf cartesische Koordinaten beziehen kann. Eine quantitative Aussage ist in 2 enthalten: Jeder Punkt $\mathrm{P}^{\prime}$ einer infinitesimalen Umgebung von $\mathrm{P}$ hat einen positiven Abstand von $\mathrm{P}$; die Homogenität läßt sich dann wie bei Riemann (1. c. ${ }^{2}$, II, Nr. l) begründen. Das Wesentliche ist im Postulat 3 enthalten. Es liegt nahe, dieses Isotropiepostulat für den Raum der Physik unter Heranziehung des Prinzips vom zu. reichenden Grunde einsichtig zu machen. Eine Anisotropie des Raumes müßte sich stets an Dingen im Raume bemerkbar machen, und man würde dann eine physikalische Ursache (etwa ein Feld) im Raume für die Erklärung der Anisotropie heranziehen.

Dissertation (1953) des Verf., Differentialgeometrie ohne Dimensionsaxiom. Wir werden hier auch von der dort durchgeführten Erweiterung des Begriffs der Riemannschen Metrik auf unendlichdimensionale Räume Gebrauch machen. 
Den komplexen linearen Zustandsraum der Quantenmechanik können wir sofort auf den reellen Fall zurückführen. Dazu denken wir uns nicht nur durch den Zustandsvektor $\psi$, sondern auch durch die $e^{i \mu} \psi, 0<\mu<\pi$, Richtungen konstituiert, und dann lassen wir nur reelle Multiplikatoren zu. Postulat 1 fällt hier mit dem Superpositionsprinzip zusammen. Postulat 2 enthält die Entscheidung darüber, welches unter den $\psi$, die denselben Zustand repräsentieren, ,normiert" ist. 3 besagt hier: Alle Zustände sind an sich gleichberechtigt. Jede Eigenschaft, die einem Zustand als solchem zukommt, trifft auch für jeden anderen zu. (Erst die Beobachtung zeichnet Zustände aus, z. B. als Eigenzustände von Observablen.)

Zunächst ist klar, daß für $F(\mathfrak{x})=\langle\mathfrak{x} \mid \mathfrak{x}\rangle^{1 / 2}(\langle\mathfrak{x} \mid \mathfrak{y}\rangle$ ein Skalarprodukt, d. h. eine Bilinearform mit positiv-definitem $\langle\mathfrak{x} \mid \mathfrak{x}\rangle)$ in einem linearen Raum beliebiger Dimension die Postulate $1-3$ erfüllt sind. Denn die Isotropie folgt sofort aus der Existenz der Drehungsgruppe, die jede Richtung unter Erhaltung aller geometrischen Eigenschaften in eine beliebige andere überzuführen vermag. Die eigentliche Aussage ist die folgende Umkehrung:

Satz. Unter den Voraussetzungen 1, 2, 3 ist $F(\mathfrak{x})=$ $\langle\mathfrak{x} \mid \mathfrak{x}\rangle^{1 / 2}$, wobei $\langle\mathfrak{x} \mid \mathfrak{x}\rangle$ eine positiv-definite quadratische Form ist.

Der Raum ist also euklidisch-metrisch bzw. hilbertsch. Daraus ergibt sich sofort für den differentialgeometrischen Fall (Postulat $\mathbf{l}^{\prime}$ mit 2, 3 für den Tangentialraum in jedem Punkte), daß es sich um einen Riemannschen Raum im klassischen Sinne oder um einen verallgemeinerten, unendlichdimensionalen Riemannschen Raum handelt?

Zum Beweise dieses Satzes bemerken wir zunächst, daß wir voraussetzen können, der Raum habe mindestens die Dimensionszahl 2; denn für einen eindimensionalen Raum ist der Satz trivial. Von nun an betrachten wir einen beliebigen, aber fürs erste festgehaltenen zweidimensionalen linearen Unterraum durch den Nullpunkt. In diesem interessieren wir uns für die Gesamtheit der Vektoren $\mathfrak{x}$ mit $F(\mathfrak{x})=1$, die wegen Postulat 2 eine den Nullpunkt nicht treffende stetige Kurve ist, welche mit jedem Halbstrahl genau einen Punkt gemeinsam hat und zum Nullpunkt symmetrisch ist.

${ }^{8}$ Konvexe Mittelpunktsbereiche und Minkowskische (normierte) Räume, erscheint demnächst in der Math. Z.
Diese Kurve möge die Indikatrix genannt werden; sie spielt in dieser Geometrie die Rolle des Einheitskreises. Die euklidischen Ebenen sind dadurch gekennzeichnet, daß in ihnen die Indikatrix eine Ellipse ist.

An anderer Stelle ${ }^{8}$ wird ein Beweis des Satzes angegeben, ohne daß irgendwelche weitere Forderungen an die Indikatrix gestellt werden. Der Kürze halber möge hier angenommen werden, daß die Indikatrix ausreichende Differenzierbarkeitseigenschaften besitzt. Aus Postulat 3 folgt, daß alle gegenüber der zentrisch-affinen Gruppe invarianten Eigenschaften der Indikatrix in einem Punkte ihr auch in jedem anderen Punkte zukommen. Die Indikatrix muß daher konvex sein. Dann gibt es Affinnormalen durch den Nullpunkt ${ }^{9}$, und nach Postulat 3 gehen dann alle Affinnormalen durch den Nullpunkt; daraus folgt aber ${ }^{9}$, daß die Indikatrix eine Ellipse ist. Der betrachtete zweidimensionale Unterraum ist also eine euklidische Ebene. Dann läßt sich aber leicht zeigen ${ }^{8}$, daß auch der ganze Raum euklidisch ist, da die betrachtete Ebene ganz beliebig gewählt war. Es gilt also $F(\mathfrak{x})=\langle\mathfrak{x} \mid \mathfrak{x}\rangle^{1 / 2}$ mit einem Skalarprodukt $\langle\mathfrak{x} \mid \mathfrak{y}\rangle$, was zu zeigen war.

Damit ist gezeigt, daß die isotropen linearen metrischen Räume die euklidischen bzw. Hilbertschen Räume sind und daß die in jedem Punkte im kleinen isotropen Räume gerade die Riemannschen Räume sind. Dann ergibt sich unmittelbar, daß ein im großen isotroper homogener Raum notwendig ein Riemannscher Raum von konstanter Krümmung ist. Ferner erhalten wir eine Auszeichnung der Weylschen Infinitesimalgeometrie ${ }^{10}$; da unsere Kennzeichnung rein infinitesimal ist, wird nämlich die Freiheit in der Wahl der Eichung nicht angetastet.

Unsere Begründung des Isotropiepostulates für den Raum der Physik beruhte wesentlich auf der an Newton anschließenden Auffassung des Raumes als eines absoluten Ordnungsschemas der physikalischen Phänomene. Dies ist im Lichte der allgemeinen Relativitätstheorie gewiß unbefriedigend, ist aber durchaus verträglich mit dem Einsteinschen Äquivalenzprinzip. Denn in einem Koordinatensystem mit lokal forttransformierter Gravitation wird auch bei Einstein noch de facto

${ }^{9} \mathrm{~W}$. Blaschke, Differentialgeometrie II, Berlin 1923, besonders S. 18 u. 47.

${ }^{10}$ H. Weyl, l. c. $^{3}$ und Raum, Zeit, Materie, 5. Aufl., Springer-Verlag, Berlin 1923. 
am Newtonschen Standpunkt festgehalten. Dem Schritt von Newton zu Einstein wird dadurch Rechnung getragen, daß die Postulate infinitesimal gefaßt werden. Weyl drückt diesen Sachverhalt so aus: In der quantitativen Ausgestaltung des metrischen Feldes besteht noch Freiheit, wenn auch die Natur der Metrik (als pythagoreische) für alle Punkte die gleiche ist und von vornherein feststeht.

Hier muß darauf hingewiesen werden, daß Weyls Behandlung des Raumproblems ohne Schwierigkeit auch die indefinite relativistische Weltmetrik erfaßt. Dagegen liegt es im Wesen unseres Isotropiepostulates, daß wir wegen des natürlichen Unterschieds zwischen raumartigen und zeitartigen Richtungen die vierdimensionale Welt nicht direkt behandeln können. Wir haben bisher nur die Riemannsche Struktur für Raum und Zeit getrennt begründen können (für die eindimensionale Zeit ist dies ja ohnehin trivial). Der Zusammenhang zwischen Raum und Zeit muß durch ein zusätzliches physikalisches Prinzip erfaßt werden, und wenn man dafür im Infinitesimalen das spezielle Relativitätsprinzip heranzieht, ergibt sich tatsächlich die indefinite quadratische Form für das Quadrat des Elements der Eigenzeit.

Die Tragweite des Isotropiesatzes dürfte über die angegebenen Beispiele (physischer Raum und quantenmechanische Zustandsmannigfaltigkeit) wohl noch hinausgehen. Immer dann, wenn in einer Gesamtheit von im Sinne der betrachteten Eigenschaften gleichberechtigten Dingen quantitative Aussagen gemacht werden, wird eine quadratische Form eine wesentliche Rolle spielen. Damit scheint es zusammenzuhängen, daß im Konfigurationsraum irgendeines klassisch-mechanischen Systems eine Riemannsche Metrik in Verbindung mit der kinetischen Energie auftritt. Entsprechendes gilt, wie näher auszuführen wäre, für das Auftreten des $\Delta$ - bzw. $\square$-Operators in den physikalischen Grundgesetzen

$$
\left(\Delta=\delta^{i k} \frac{\partial}{\partial x_{i}} \frac{\partial}{\partial x_{k}}, \square=g^{\alpha \beta} \frac{\partial^{*}}{\partial x^{\alpha}} \frac{\partial^{*}}{\partial x^{\beta}}\right) .
$$

Es sei uns noch ein kurzer Blick in das Gebiet einer Nachbarwissenschaft, der Erkenntnistheorie, erlaubt. Manche Autoren stellen dem physischen Raum noch einen Anschauungsraum als Ordnungsschema unserer Sinneseindrücke zur Seite. Daß dieser a priori isotrop

11 Über die Rolle des Prinzips vom zureichenden Grunde in der Physik vergleiche man H. Weyl, Philosophie der Mathematik und Naturwissenschaft sei, wird man zugeben können. Anisotropien würden wieder nach dem Prinzip vom zureichenden Grunde nicht dem Raume, sondern den Dingen in ihm zugeschrieben werden. Dann folgt aber vermöge unseres Satzes die Euklidizität des Anschauungsraumes, sobald die Vergleichbarkeit von Linien in bezug auf ihre Länge zugelassen wird.

An dieser Stelle mag auch bemerkt werden, daß die gelegentlichen Anwendungen, die das Prinzip vom zureichenden Grunde in der Physik bei der Auffindung von Naturgesetzen erlaubt ${ }^{11}$, gewöhnlich gruppentheoretische Eigenschaften der euklidischen Geometrie bereits voraussetzen. (So braucht Archimedes zur Herleitung der Hebelgesetze die Spiegelung.) Wenn wir hier dieses Prinzip zur Begründung des Isotropiepostulates herangezogen haben, so ist dabei nichts Gruppentheoretisches vorausgesetzt worden. Freilich folgt nachher mit der Euklidizität die Existenz der orthogonalen Gruppe.

Schließlich sei noch auf einige offene Fragen hingewiesen.

Im Zustandsraum haben wir das Superpositionsprinzip von vornherein vorausgesetzt. Man kann fragen, ob sich dieses Prinzip nicht seinerseits auch aus der Forderung der Gleichberechtigung aller Zustände vor der Beobachtung ableiten läßt. Wir wollen noch einen vielleicht gangbaren Weg dafür andeuten. Wenn man von der Gesamtheit der $\mathrm{Zu}$ stände noch weiß, wann zwei Zustände ,,benachbart" sind, dann läßt sich die Menge der Zustände mathematisch als topologischer Raum auffassen. Wenn sich dieser Konvergenzbegriff wenigstens für kleine Umgebungen jedes Zustands durch eine Abstandsdefinition darstellen läßt und wenn man dann noch den Zustandsraum im kleinen auf Koordinaten aus einem linearen Raum beziehen kann, so wie man endlich dimensionale Räume auf cartesische oder Gaußsche Koordinaten bezieht, dann darf man aus der Forderung, daß von einem $\mathrm{Zu}$ stand aus keine Richtung zu einem benachbarten Zustand vor anderen ausgezeichnet sein soll, mit Hilfe des Isotropiesatzes sofort schließen : Der Zustandsraum ist ein Riemannscher Raum, über dessen Dimension freilich nichts gesagt ist (sie darf auch gleich einer unendlichen Kardinalzahl sein). Aus der Gleichberechtigung aller Zustände folgt zunächst die Konstanz des Riemannschen

(Hdb. d. Phil.), München 1928, S. 121 und E. Mach, Die Mechanik in ihrer Entwicklung, 4. Aufl., Leipzig 1901, S. 12. 
Krümmungsmaßes. Dann wird man zeigen müssen, daß man diesen Riemannschen Raum konstanter Krümmung durch eine Kugel $\langle\mathfrak{x} \mid \mathfrak{x}\rangle=1$ in einem euklidischen (Hilbertschen) Raum darstellen kann. Wir können uns dann die Zustände wie üblich durch die Strahlen in diesem linearen Raum repräsentiert denken und haben außer der quadratischen Metrik auch die Möglichkeit der Superposition erhalten. Eine genauere Durchführung dieses Gedankenganges würde eine bessere als die heute vorhandene Kenntnis der Differentialgeometrie der unendlichdimensionalen Räume erfordern. (Man vergleiche die zitierte Arbeit des Verfassers $^{7}$ und die dort angegebene Literatur.) Ein prinzipiell nicht einfacherer, aber mehr formaler und deshalb kürzerer Weg zur Lösung dieses Problems könnte der folgende sein. Jeder Zustand $Z$ soll repräsentiert gedacht werden durch die Gesamtheit der Paare $\lambda Z$ mit von Null verschiedenen Zahlen $\lambda$. Ferner identifizieren wir alle Paare $0 Z$ und schreiben dafür 0. Eine Topologie in dieser neuen Mannigfaltigkeit wird dann mit einer Topologie in der Menge der $\lambda Z$ in folgender Weise gegeben sein:

$$
\begin{aligned}
& \lambda_{n} Z_{n} \rightarrow \lambda Z, \text { wenn } \lambda_{n} \rightarrow \lambda \text { und } Z_{n} \rightarrow Z, \\
& \lambda_{n} Z_{n} \rightarrow 0, \text { wenn } \lambda_{n} \rightarrow 0 .
\end{aligned}
$$

Können wir jetzt in einer Umgebung von $0 \mathrm{Ko}$ ordinaten aus einem linearen Raume einführen und definieren wir $F(\lambda Z)=|\lambda|$, so erhalten wir aus der Gleichberechtigung aller Zustände vermöge des Isotropiesatzes, da $\beta$ die Menge der $\lambda Z$ ein linearer, Hilbertscher Raum ist.

Es kommt dabei offenbar wesentlich darauf an, in einem Hausdorffschen topologischen Raum mit lauter gleichberechtigten Punkten lineare Koordinaten einzuführen. Dies ist jedenfalls eine schwächere Annahme als die Linearität des Raumes selbst, ihr mathematischer Beweis für solche ,,topologisch homogenen" Räume steht aber aus. Die Richtigkeit der Annahme läßt sich durch Betrachtung stetiger reellwertiger Funktionen über dem Raume wenigstens plausibel machen, ähnlich wie Riemann es in seinem Habilitationsvortrag für $n$-dimensionale Räume getan hat.

v. Weizsäcker hat darauf hingewiesen, daß die ganze Erörterung noch nicht verständlich mache, warum für die Multiplikatoren gerade die komplexen Zahlen in Frage kommen. Dieses Problem sei den offenen Fragen noch angereiht.

Herrn Prof. C. F. v. Weizsäcker bin ich für Anregungen und Hinweise zu großem Dank verpflichtet. Mein Dank gilt auch Herrn Prof. G. Lyra für Gespräche, die zur Klärung der Gedanken beitrugen.

Anmerkung. Der Verfasser ist verschiedentlich gefragt worden, warum aus der Isotropievoraussetzung für $\mathrm{d} s^{2}=g_{i k}(x ; \mathrm{d} x) \mathrm{d} x^{i} \mathrm{~d} x^{k}$ nicht direkt die Unabhängigkeit des Tensors $g_{i k}$ von der Richtung $\mathrm{d} x$ geschlossen werden kann. Das gleiche Argument würde aber im Ansatz $|\mathrm{d} s|=\left|g_{i}(x ; \mathrm{d} x) \mathrm{d} x^{i}\right|$ zu dem offenbar unsinnigen Resultat $g_{i}(x ; \mathrm{d} x)=g_{i}(x)$ führen. Tatsächlich kann der Beweis also nicht durch dieses formale Argument geführt werden, welches sich zudem nicht direkt aus der hier formulierten Isotropievoraussetzung 3 begründen läßt. 


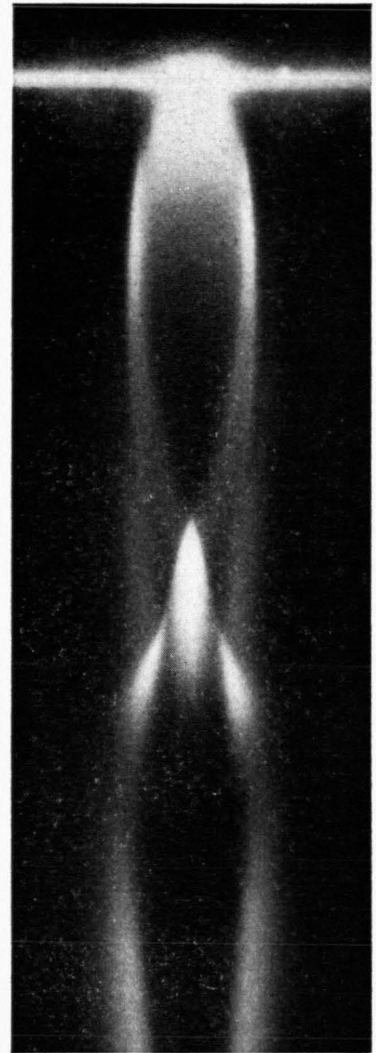

Abb. 2. Gasstrahl (Ar $+8 \%$ $\mathrm{N}_{2}$ ) aus einer Laval-Düse. Abmessungen der Düse:

Kleinster Durchmesser $0,35 \mathrm{~mm}$, Mündungsdurchmesser 1,3 mm, Länge $6 \mathrm{~mm}$. Die Düsenmündung befindet sich am oberen Bildrand.

$p_{1}=600$ Torr $; p_{\mathrm{m}}=0,5$ Torr. Vergr. 3,9-fach.

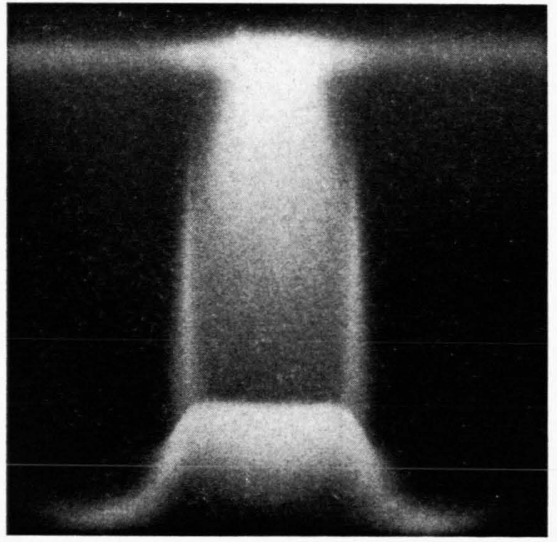

Abb. 5. Verdichtungsstoß vor einer ebenen Wand in einer Strömung nach Abb.2. Die Wand befindet sich am unteren Bildrand. $p_{1}=600$ Torr; $p_{\mathrm{m}}=0,5$ Torr. Vergr. 7,7-fach.

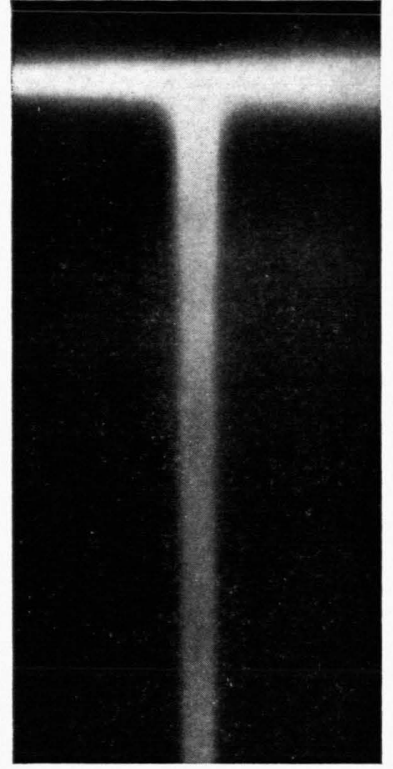

Abb. 3. Gasstrahl, bestehend aus $\mathrm{N}_{2}$. Anordnung wie in Abb. 2.

$p_{1}=600$ Torr $; p_{\mathrm{m}}=10$ Torr. Vergr. 3,9-fach.

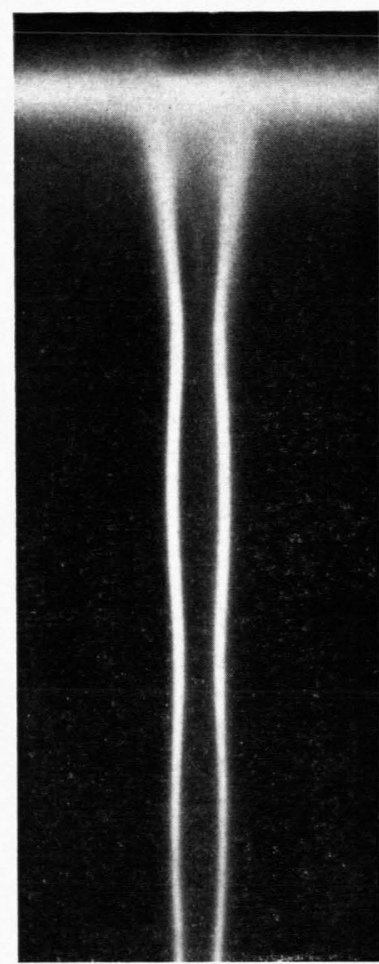

Abb. 6. Angeregter ArStrahl in CO-Atmosphäre. Laval-Düse $0,5 \times 2 \times 15 \mathrm{~mm}$. $p_{1}=600$ Torr $; p_{\mathrm{m}}=5$ Torr Vergr. 3,9-fach.

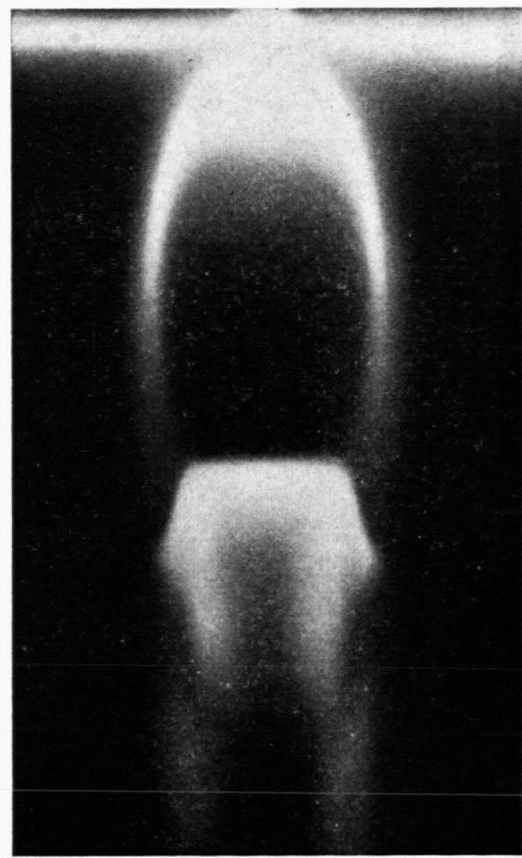

Abb. 4. Gasströmung ( $\left.\mathrm{Ar}+8 \% \mathrm{~N}_{2}\right)$ aus einer zylindrischen Düse von $0,6 \mathrm{~mm}$ Durchmesser und $2,7 \mathrm{~mm}$ Länge. Die Düsenmündung befindet sich am oberen Bildrand. $p_{1}=600$ Torr ; $p_{\mathrm{m}}=0,5$ Torr. Vergr. 4,6-fach. 


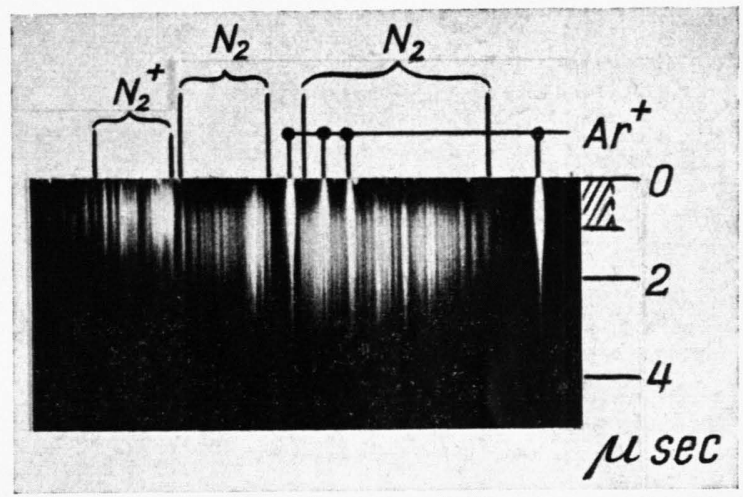

Abb. 8. Ausschnitt aus dem Spektrochronogramm des Gemischs $\mathrm{Ar}+8 \% \mathrm{~N}_{2}$. Der Ort des Elektronenbündels und der Zeitmaßstab sind am rechten Bildrand angegeben. $p_{\mathrm{m}}=0,5$ Torr.

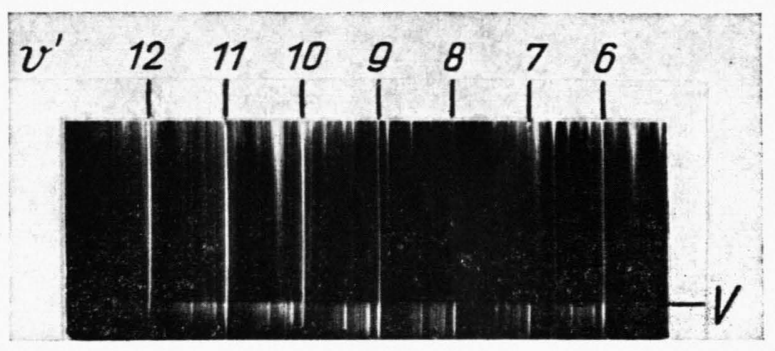

Abb. 10. Erhöhung der Helligkeit des Nachleuchtens durch Dichteerhöhung hinter einem Verdichtungsstoß nach Abb. 5. Dargestellt ist dieselbe Bandengruppe wie in Abb. 9 rechts. Der Ort des Verdichtungsstoßes $(\mathrm{V})$ ist am rechten Bildrand angegeben. $p_{\mathrm{m}}=0,5$ Torr.

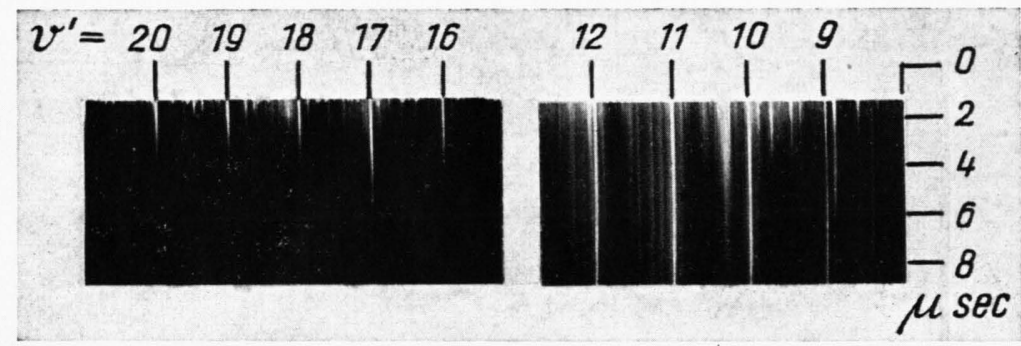

Abb. 9. Einige Banden der ,,1. positiven Gruppe“ des $\mathrm{N}_{2}$ im Nachleuchten des Gemischs $\mathrm{Ar}+8 \% \mathrm{~N}_{2}$. (Sichtbar sind nur die Bandenköpfe.) $p_{\mathrm{m}}=0,5$ Torr.

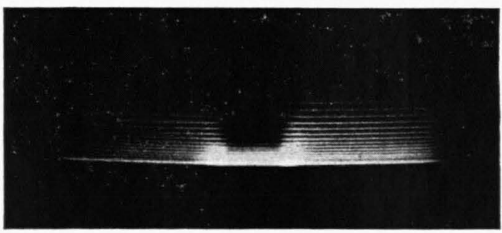

Abb. 11. Zur baridenspektroskopischen Temperaturmessung im Gasstrahl. Dargestellt ist die Bande $(0,3)$ des Ångströmsystems in CO. $p_{\mathrm{m}}=0,5$ Torr

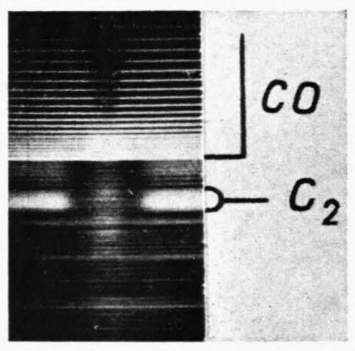

Abb. 12. ,Hochdruck-Bande“" $(6,5)$ des $\mathrm{C}_{2}$ im ruhenden und strömenden CO. $p_{\mathrm{m}}=8$ Torr. 


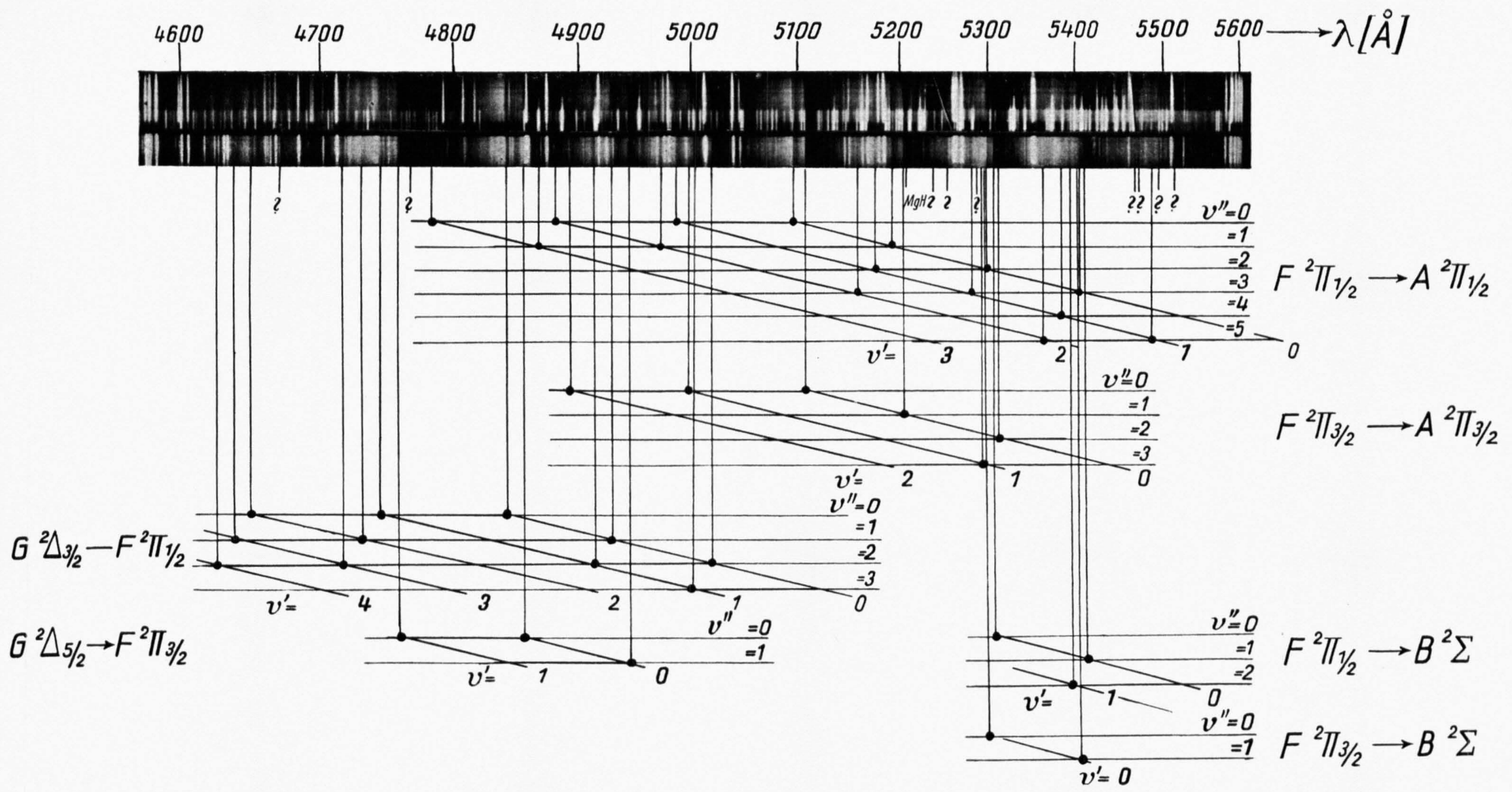

Abb. 2. Das CaCl-Spektrum zwischen $\lambda=4600$ und $\lambda=5600 \AA$. 

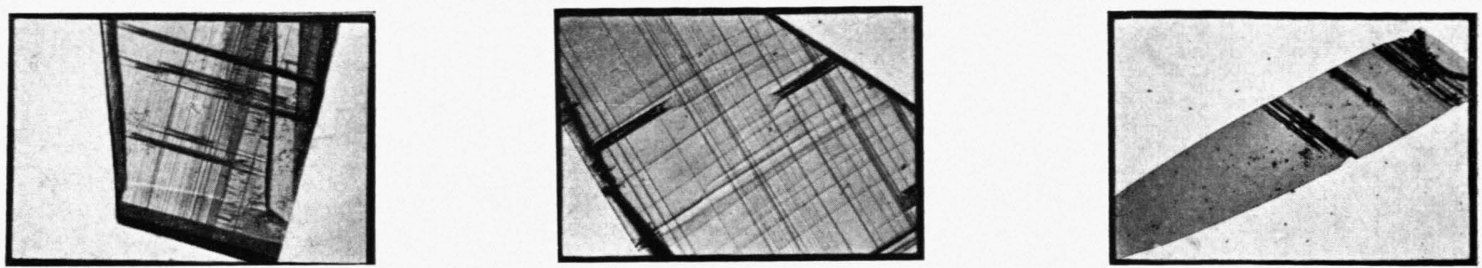

Abb. 1-3. Nach Umsublimation entstandene ZnS-Kristalle.

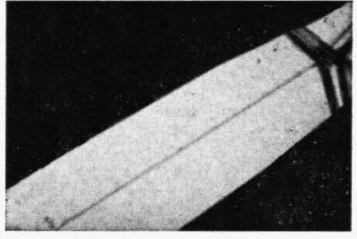

Abb. 5. ZnS-Zwillingskristall im Durchlicht.
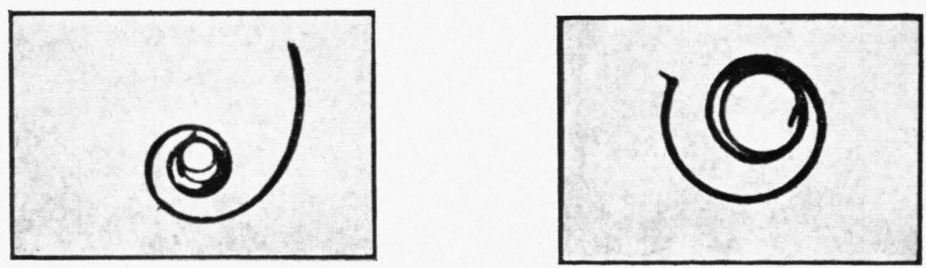

Abb. 8-9. Spiraliges Kristallwachstum von $\mathrm{ZnS}$-Kristallen.

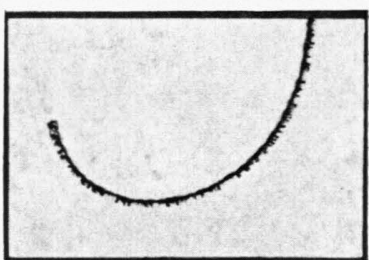

Abb. 10. Vermutlicher Beginn eines spiraligen Kristallwachstums. 